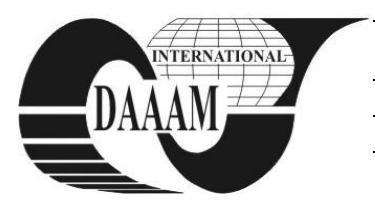

Annals of DAAAM for 2012 \& Proceedings of the 23rd International DAAAM Symposium, Volume 23, No.1, ISSN 2304-1382 ISBN 978-3-901509-91-9, CDROM version, Ed. B. Katalinic, Published by DAAAM International, Vienna, Austria, EU, 2012 Make Harmony between Technology and Nature, and Your Mind will Fly Free as a Bird Annals \& Proceedings of DAAAM International 2012

\title{
VEHICLE STEERING DYNAMIC CALCULATION AND SIMULATION
}

\author{
VU, T[rieu] M[inh]
}

\begin{abstract}
This paper presents fundamental mathematical estimations of vehicle sideslip in stationary conditions regarding the influences of the vehicle parameters such as the tire stiffness, the position of gravity center, the vehicle speed and the turning radius. The vehicle dynamics on steady state and transient responses are also investigated to see the effects of the yaw natural frequency and yaw damping rate on the steering system. Results from this study can be used in designing an automatic control of tracking vehicle in the future. Keywords: sideslip angle, yaw damping rate, steady state response, transient response
\end{abstract}

\section{INTRODUCTION}

Calculation and simulation of vehicle steering dynamic are essential for any control systems since most of the modern vehicles are currently equipped with new electronic stability and auto-guided systems. The accurate determination of the sideslip angle can help to improve the yaw and the steering stability performance. Sideslip estimation is based on the vehicle physical variables (mass, gravity position, tire stiffness), vehicle speed, lateral acceleration, steering angle, and yaw rate. Unlike yaw rate, the vehicle sideslip angle cannot be measured directly; hence estimation methods have been developed to calculate the sideslip angle from the available above variables.

Among the latest research papers on this issue, Kim H. and Ryu J. in [1] have proposed a sideslip angle estimation method that considers severe longitudinal velocity variation over the short period of time based on extended Kalman filter (EKF). Hac A, el al. in [2] have established an estimation method of vehicle roll angle, lateral velocity and sideslip angle. Only roll rate sensor and the sensors readily in electronic stability control (ESC) are used in this estimation process. Mathematical algorithms are based on kinematic relationships, and then, avoiding dependence on vehicle and tire models, which can minimize tuning efforts and sensitivity to parameter variations. Lenain R., et al. in [3] introduce an observer for dynamic sideslip angle with mixed kinematic for accurate control of fast off-road mobile robots. With respect to pure kinematic approaches, the use of this dynamic representation for estimation of the sideslip angle improves reactivity in sliding variable adaptation and consequently in path tracking accuracy.

The content of this paper is mostly based on the publication in [4] on handling model of advanced vehicle dynamics where mathematical algorithms for a single track vehicle are modeled regarding the effects of the vehicle center of gravity, the front/rear tire stiffness and the under-steering/over-steering conditions. Other knowledge for yaw damping and steering control is referred on Ackermann J. and Sienel W. in [5] where a steering control system is studied with yaw damping rate and yaw natural frequency to control the unexpected yaw motions. Mathematical formulas and simulation for the vehicle models are derived from Minh V.T and Aziz A.R in [6] and from Minh V.T and Pumwa J. in [7]. Adaptive EKF-based estimation for vehicle sideslip angle can be referred to by Hrgetic $\mathrm{M}$ el. al. in [8].

This study is the first part of a project on automatic control of tracking vehicles where the vehicle sideslip is modeled and estimated. The accurate mathematical model for vehicle sideslip is critically needed for designing a free-error feedback control system for automatic trajectory tracking vehicles.

The outline of this paper is as follows: Section 2 provides fundamental mathematic formulas for calculation of vehicle sideslip; Section 3 presents the vehicle behavior with steering in steady state condition; Section 4 demonstrates the vehicle movement in transient responses and section 5 analyses the vehicle dynamic responses with frequency input; Finally, conclusion is withdrawn in section 6 .

\section{VEHICLE SIDESLIP CALCULATION}

When the vehicle is moving straight on a flat surface, the direction of the center of gravity (CG) keeps the same with the orientation of the vehicle. When the vehicle turns, the yaw rate causes the change of the orientation.

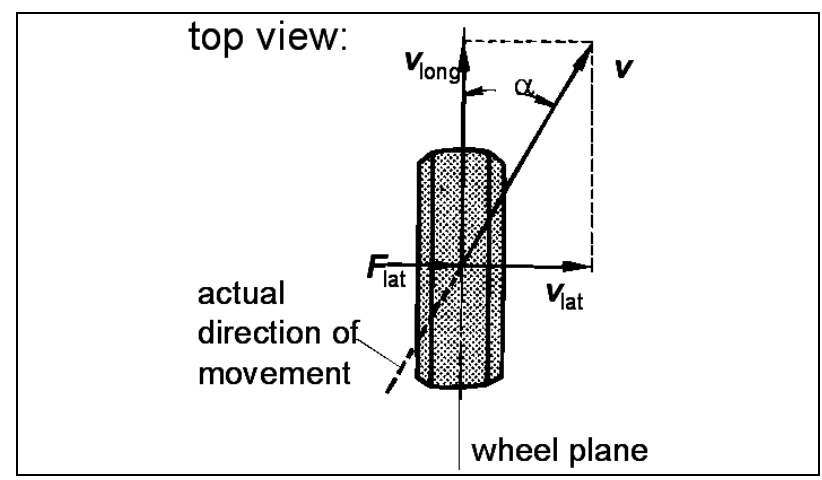

Fig. 1. Sideslip angle $\alpha$

The vehicle demonstrates a velocity component perpendicular to the orientation, known as the lateral velocity. Then, the orientation of the vehicle and the direction of the travel are no longer the same. The vehicle is moving under the influence of different forces. If a lateral force is acting on the tire, an angle is formed between the direction of movement of the tire and the tire 
straight line. This angle is called the sideslip angle $\alpha$ (shown in Fig. 1).

Reason for this sideslip angle or the tire slip is the elastic lateral deflection of the rolling tire in the tire contact area under the effect of the lateral force between tire and road.

For analyzing the motion behaviors of a single track model, a linearization of the tire lateral force and the tire slip angle is assumed via a tire stiffness $c_{\alpha}$ :

$$
c_{\alpha}=\frac{F_{\alpha}}{\alpha}
$$

When the vehicle moves at low speed, the wheels roll without a tire slip angle since the lateral cornering force, $F_{\alpha}$, is small and can be ignored. The vehicle model can be seen as the assumption of Rudolf Ackermann with the elongations of all wheel center lines intersecting at one point, the center of the turning curve (Fig. 2).

The steering angle, $\delta$, can be simply calculated as:

$$
\delta=\arctan \left(\frac{l}{r}\right)
$$

The steering angle of the inner wheel, $\delta_{i}$, is a function of the steering angle of the outer wheel, $\delta_{o}$ :

$$
\delta_{i}=\arctan \left(\frac{l}{\frac{l}{\tan \delta_{o}}-s}\right)
$$

And as a result of Ackermann condition, the angle of the inner wheel, $\delta_{i}$, is greater than the steering angle at the outer wheel, $\delta_{o}$.

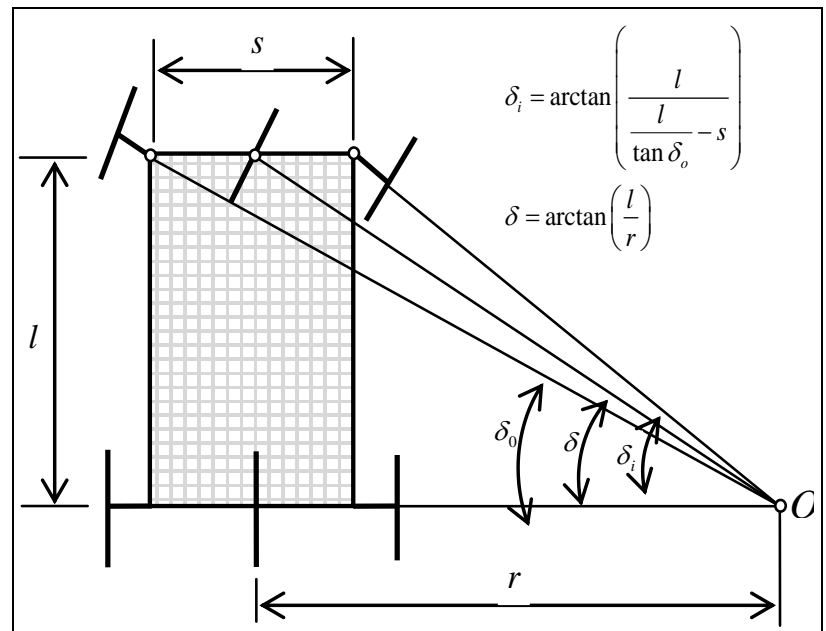

Fig. 2. Model for sideslip (Ackermann condition)

where, $\delta_{i}, \delta_{o}$ : steering angle of inner and outer wheel, ${ }^{l}$ : wheel base, $s$ : kingpin track width, $r$ : radius of the curve

However, when the lateral force appears, the vehicle front wheel orientation and the vehicle movement direction is no longer the same. A simplified description of the vehicle lateral dynamics is demonstrated in a single track model (Fig. 3). The tire contact points are in the center of tires. Longitudinal forces in the tire contact points as well as wheel load fluctuations are not considered. The height of the center of gravity is zero.
The Newton's law equation of the motion for the vehicle lateral direction is:

$$
m a_{y}=F_{L f}+F_{L r}
$$

The force of inertia acting on the vehicle center of gravity, $m a_{y}$, corresponds to the centrifugal force:

$$
m a_{y}=m \frac{v^{2}}{r}=m \frac{v}{r} \dot{v} r=m v(\dot{\Psi}-\dot{\beta})
$$

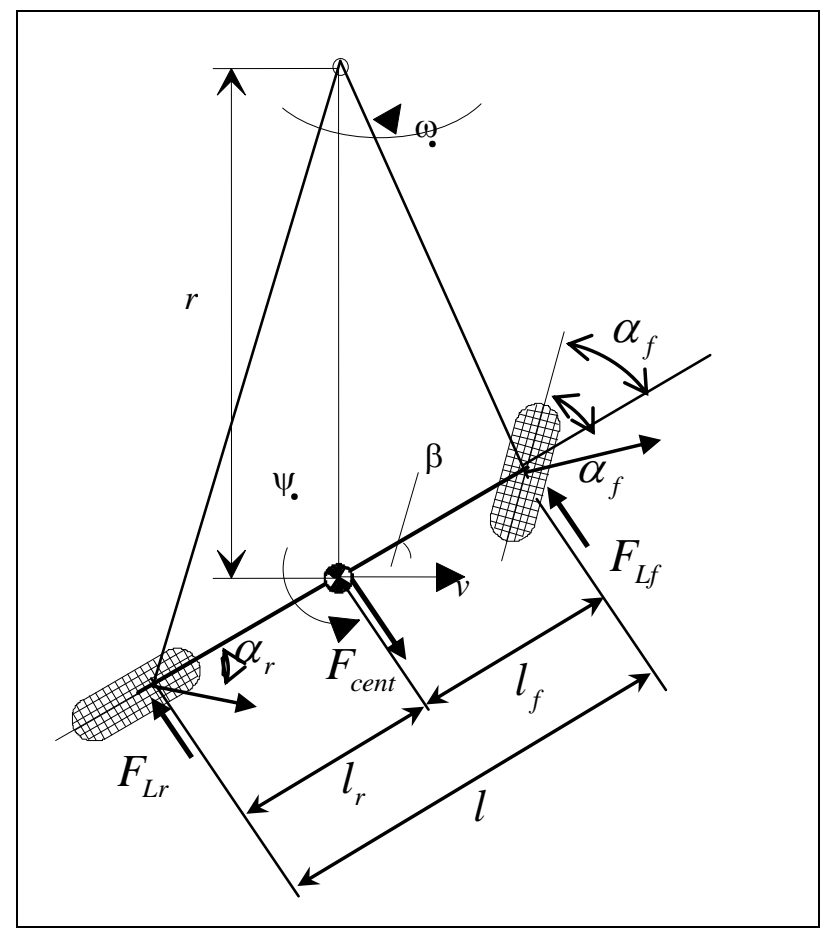

Fig. 3. Deflection of the rolling tire by a lateral cornering force $F_{\alpha}$ where, $v$ : Vehicle velocity, $\omega$ : Vehicle angular velocity, $r$ : radius of curve, $\psi$ : Yaw angle, $\beta$ : Side slip angle, $\delta:$ Steering angle, $\alpha$ : Tire slip angle, $l:$ Wheel base

And the gyroscopic effect on the z-axis at the vehicle center of gravity:

$$
J \ddot{\psi}=F_{L f} l_{f}-F_{L r} l_{r}
$$

where $J$ is the vehicle moment of inertia on z-axis.

The tire side forces can be calculated from the given tire slip rigidity, $c_{\alpha}$, in equation (1) for the front wheel:

$$
F_{L f}=c_{\alpha f} \alpha_{f}
$$

and for the rear wheel:

$$
F_{L r}=c_{\alpha r} \alpha_{r}
$$

The side slip angle for the vehicle at the center of gravity, $\beta$, can be formulated from the front tire slip, $\alpha_{f}$ :

$$
\alpha_{f}=\delta+\beta-\frac{l_{f} \dot{\Psi}}{v}
$$


and from the rear tire slip, $\alpha_{r}$ :

$$
\alpha_{r}=\beta+\frac{l_{r} \dot{\Psi}}{v}
$$

It is noted that the tire slip rigidity or the sideslip stiffness, $c_{\alpha}$, is an elastic property for each rubber tires, normally in the range of 30,000-50,000 N/rad.

\section{STEERING IN STEADY STATE}

In steady state condition, the vehicle speed, $v$, is a constant, then, the yaw velocity, $\dot{\Psi}$, and the sideslip, $\beta$, are also constant, i.e, $\ddot{\Psi}=0$ and $\dot{\beta}=0$.

The torque balance equations can be formulated at the rear contact point:

$$
F_{L f} l=m a_{y} l_{r}
$$

Replaced with the tire slip rigidity in equation (7):

$$
c_{\alpha f}\left(\delta+\beta-\frac{l_{f} \dot{\Psi}}{v}\right)=\frac{l_{r}}{l} m a_{y}
$$

And in equation (8):

$$
c_{\alpha r}\left(\beta+\frac{l_{r} \dot{\Psi}}{v}\right)=\frac{l_{f}}{l} m a_{y}
$$

Because in the steady state, $\dot{\beta}=0$, then from equation (5), $\dot{\Psi}=\frac{v}{r}$. The transformation from equation (13) and (14) leads to:

$$
\delta=\frac{l}{r}+\frac{m}{l}\left(\frac{l_{r}}{c_{\alpha f}}-\frac{l_{f}}{c_{\alpha r}}\right) a_{y}
$$

From the above equation, the necessary steering angle, $\delta$, during the steady state driving along a curve composes of two parts. The first part, $\frac{l}{r}$, or Ackermann angle, depends on the vehicle geometrical parameters. And the second part, $\frac{m}{l}\left(\frac{l_{r}}{c_{\alpha f}}-\frac{l_{f}}{c_{\alpha r}}\right) a_{y}$, is characterized by the influences of the lateral acceleration and the tire rigidities, which can increase, if $\left(\frac{l_{r}}{c_{\alpha f}}>\frac{l_{f}}{c_{\alpha r}}\right)$, or reduce, if

$$
\left(\frac{l_{r}}{c_{\alpha f}}<\frac{l_{f}}{c_{\alpha r}}\right) \text {, the steering angle. }
$$

From equation (9) and (10), the sideslip angle difference between the front and the rear wheel is:

$$
\Delta \alpha=\alpha_{f}-\alpha_{r}=\delta-\frac{l \dot{\Psi}}{v}
$$

With $v=\dot{\Psi} r$, then, $\Delta \alpha=\delta-\frac{l}{r}$. Replace with $\delta$ in equation (14):

$$
\Delta \alpha=\frac{m}{l}\left(\frac{l_{f}}{c_{\alpha r}}-\frac{l_{r}}{c_{\alpha f}}\right) a_{y}
$$

Then, the difference of the sideslip angles depends on the vehicle and the tire parameters. The driver has to compensate the sideslip angle difference, $\Delta \alpha$, with the steering angle, $\delta$. This forms a basic knowledge of oversteer and under-steer definition: Over-steer is if $\Delta \alpha=\alpha_{f}-\alpha_{r}<0$, neutral is if $\Delta \alpha=\alpha_{f}-\alpha_{r}=0$, and under-steer is if $\Delta \alpha=\alpha_{f}-\alpha_{r}>0$ (Fig. 4).

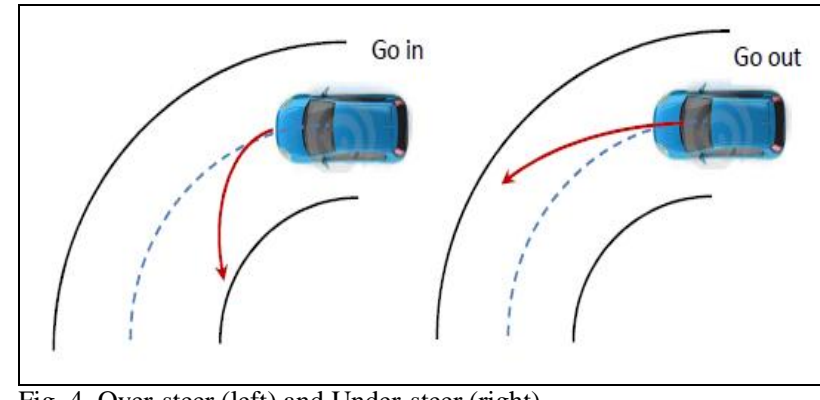

Fig. 4. Over-steer (left) and Under-steer (right)

Under-steer and over-steer are vehicle dynamic characteristics used to demonstrate the sensitivity of a vehicle steering system. The under-steer happens if the vehicle turns less than the steering control of the driver. Conversely, over-steer happens if the vehicle turns more than the steering control of the driver.

The under-steer system is safer since it causes the reduction of the lateral force at the rear axle and makes the vehicle to stabilize at a smaller curve radius with less lateral acceleration. While the over-steer vehicle is more dangerous because it increases the lateral force and increases the swerve tendency of the vehicle.

Fig. 5 demonstrates the relationship between the sideslip and steering angle with the vehicle speed and the turning radius. When maintaining the turning radius at $r=100 \mathrm{~m}$ and varying the vehicle speeds, $0 \leq v \leq 40 \mathrm{~m} / \mathrm{s}$, the sideslips increase exponentially and steering angles rise, $1.4^{0} \leq \delta \leq 3.2^{0}$. Similarly, when reducing the turning radius $100 \leq r \leq 10 \mathrm{~m}$, the sideslips increase exponentially and steering angles rise $2^{0} \leq \delta \leq 20^{\circ}$.

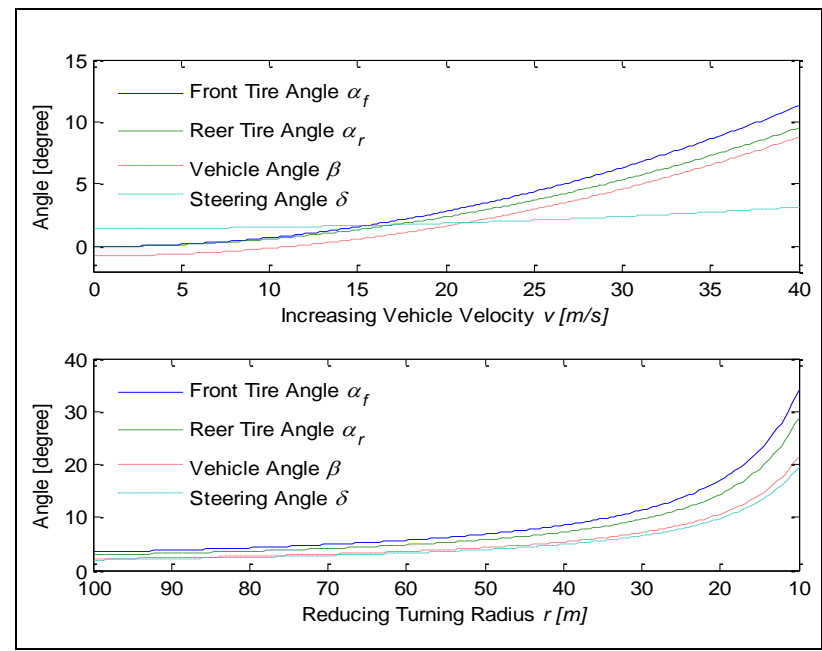

Fig. 5. Side and steering angle vs. velocity and turning radius 


\section{STEERING IN TRANSIENT RESPONSE}

Transformation of equations (4-10) can lead to the following expressions:

$$
m v(\dot{\psi}-\dot{\beta})=c_{\alpha f}\left(\delta+\beta-\frac{l_{f}}{v} \dot{\psi}\right)+c_{\alpha r}\left(\beta+\frac{l_{r}}{v} \dot{\psi}\right)
$$

and

$$
J_{z} \ddot{\psi}=c_{\alpha f}\left(\delta+\beta-\frac{l_{f}}{v} \dot{\psi}\right) l_{f}-c_{\alpha r}\left(\beta+\frac{l_{r}}{v} \dot{\psi}\right) l_{r}
$$

Equation (17) can be represented by yaw velocity, $\dot{\psi}$ :

$$
\dot{\psi}=\frac{m v \dot{\beta}+c_{\alpha f}(\delta+\beta)+c_{\alpha r} \beta}{m v-c_{\alpha r} \frac{l_{f}}{v}+c_{\alpha f} \frac{l_{r}}{v}}
$$

For the steady state, $v=$ const, then, $\ddot{\psi}$ :

$$
\ddot{\psi}=\frac{m v \ddot{\beta}+c_{\alpha f}(\dot{\delta}+\dot{\beta})+c_{\alpha r} \dot{\beta}}{m v-c_{\alpha r} \frac{l_{r}}{v}+c_{\alpha f} \frac{l_{f}}{v}}
$$

Replace $\dot{\psi}$ and $\ddot{\psi}$ in equation (18):

$$
\begin{gathered}
\ddot{\beta}+\left(\frac{c_{\alpha f}+c_{\alpha r}}{m v}+\frac{c_{\alpha f} l_{f}^{2}+c_{\alpha r} l_{r}^{2}}{v J_{Z}}\right) \dot{\beta} \\
+\left(\frac{c_{\alpha r} l_{r}-c_{\alpha f} l_{f}}{J_{Z}}+\frac{c_{\alpha f} c_{\alpha r} l^{2}}{J_{Z} m v^{2}}\right) \beta \\
=\left(\frac{c_{\alpha f} l_{f}}{J_{Z}}-\frac{c_{\alpha f} c_{\alpha r}\left(l_{f} l_{r}+l_{r}^{2}\right)}{J_{Z} m v^{2}}\right) \delta-\frac{c_{\alpha f}}{m v} \dot{\delta}
\end{gathered}
$$

The characteristic polynomial of the dynamic equation in (21) can be represented in an inhomogeneous linear differential equation of $2^{\text {nd }}$ order for the vehicle slip angle $\beta$. The homogeneous part of this differential equation has the form of a simple oscillating motion with damping:

$$
\ddot{\beta}+A \dot{\beta}+B \beta=0
$$

or in the vibrated frequency form:

$$
\omega_{0}^{2}+2 D \omega_{0} s+s^{2}=0
$$

Thus, the differential equation for the slip angle $\beta$ can be viewed with a yaw undamped natural frequency, $\omega_{0}$ :

$\omega_{0}=\sqrt{\frac{c_{\alpha r} l_{r}-c_{\alpha f} l_{f}}{J_{Z}}+\frac{c_{\alpha f} c_{\alpha r} l^{2}}{J_{Z} m v^{2}}}$

and with a yaw damping rate $D$ :

$$
D=\frac{\frac{c_{\alpha f}+c_{\alpha r}}{m v}+\frac{c_{\alpha f} l_{f}^{2}+c_{\alpha r} l_{r}^{2}}{J_{Z} v}}{2 \omega_{0}}
$$

Then, the dynamic yaw frequency, $\omega_{o m D}$, is:

$$
\omega_{0 m D}=\sqrt{1-D^{2}} \omega_{0}
$$

The yaw natural frequency and damping rate can be represented for the movement of the vehicle around the vertical axis $(\mathrm{z})$.

\section{VEHICLE DYNAMIC ANALYSIS}

The linearized single track vehicle model is now examined under the reaction of the driver to control the vehicle movement with the input variable, the steering angle, $\delta$. The output variables are the yaw velocity, $\dot{\psi}$, and the lateral acceleration, $a_{y}$.

The transfer function of the output, $\dot{\psi}$, and the input, $\delta$ can be derived from equation (14) with $\frac{1}{r}=\frac{\dot{\psi}}{v}$ and $a_{y}=v \dot{\psi}$, then:

$$
\left(\frac{\dot{\psi}}{\delta}\right)_{s t a t}=\frac{v}{l+\frac{m}{l}\left(\frac{l_{r}}{c_{s f}}-\frac{l_{f}}{c_{s r}}\right) v^{2}}
$$

where the relation $\left(\frac{\dot{\psi}}{\delta}\right)_{\text {stat }}$ is referred to as the stationary yaw amplification factor.

For analyzing the dynamic behavior of the vehicle, equations (17) and (18) can be converted to Laplace $s$ form

$$
\frac{\dot{\psi}}{\delta}=F(s)=\left(\frac{\dot{\psi}}{\delta}\right)_{\text {stat }} \frac{1+T_{z} s}{1+\frac{2 D}{\omega_{0}} s+\frac{1}{\omega_{0}^{2}} s^{2}}
$$

with $T_{z}$ is a time constant, $T_{z}=\frac{m v l_{f}}{c_{\alpha r} l}$.

This Laplace $s$-form can also be transformed for the lateral acceleration:

$$
\frac{a_{y}}{\delta}=F^{\prime}(s)=\left(\frac{a_{y}}{\delta}\right)_{\text {stat }} \frac{1+T_{1} s+T_{2} s^{2}}{\frac{2 D}{\omega_{0}} s+\frac{1}{\omega_{0}^{2}} s^{2}}
$$

with the time constant $T_{1}=\frac{l_{r}}{v}$, and $T_{2}=\frac{J_{Z}}{c_{\alpha r} l}$.

Simulations for the driving control of the vehicle movement are conducted with a step steering (sudden step in input signal) and shown in Fig. 6.
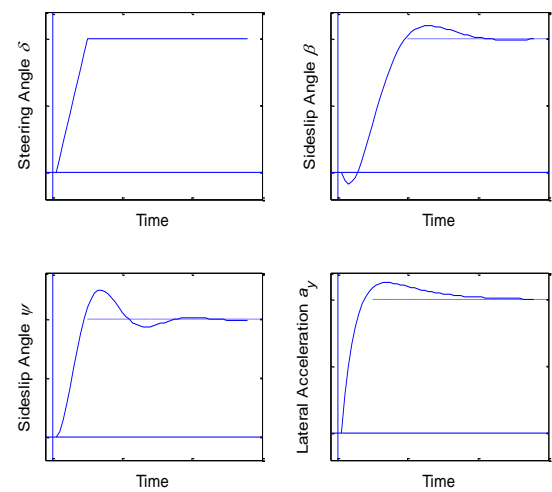

Fig. 6. Transient response with a steering step angle 
For a very fast input of a steering step angle to $20^{\circ}$ in 0.4 second, the sideslip angle, $\psi$, and the lateral acceleration, $a_{y}$, respond with a small overshooting motion and then steadily fluctuate at the stable position; While the sideslip angle, $\beta$, responds in an undershooting motion at the beginning time.

For frequency response, the transfer function in equation (28) now is transformed into the frequency, $j \omega$ , form:

$$
F(j \omega)=\left(\frac{\dot{\psi}}{\delta}\right)_{\text {stat. }} \frac{1+T_{z} j \omega}{1+\frac{2 \cdot D}{\omega_{0}} j \omega-\frac{\omega^{2}}{\omega_{0}^{2}}}
$$

The amplitude, $\left(\frac{\hat{\dot{\psi}}}{\hat{\delta}}\right)=|F(j \omega)|$, is thus a frequency dependence.

The lateral acceleration in equation (29) is now applied for the frequency response:

$$
F^{\prime}(j \omega)=\left(\frac{a_{y}}{\delta}\right)_{\text {stat. }} \frac{1+T_{1} j \omega-T_{2} \cdot \omega^{2}}{1+\frac{2 \cdot D}{\omega_{0}} j \omega-\frac{\omega^{2}}{\omega_{0}^{2}}}
$$

Simulation results of frequency response are shown in Fig. 7. There is a peak of magnitude and phase shifting in the low frequencies. The amplitude responses drop in high frequencies. There is a phase lag in yaw velocity and thus, the vehicle reaction on the steering angle becomes larger in low frequencies.

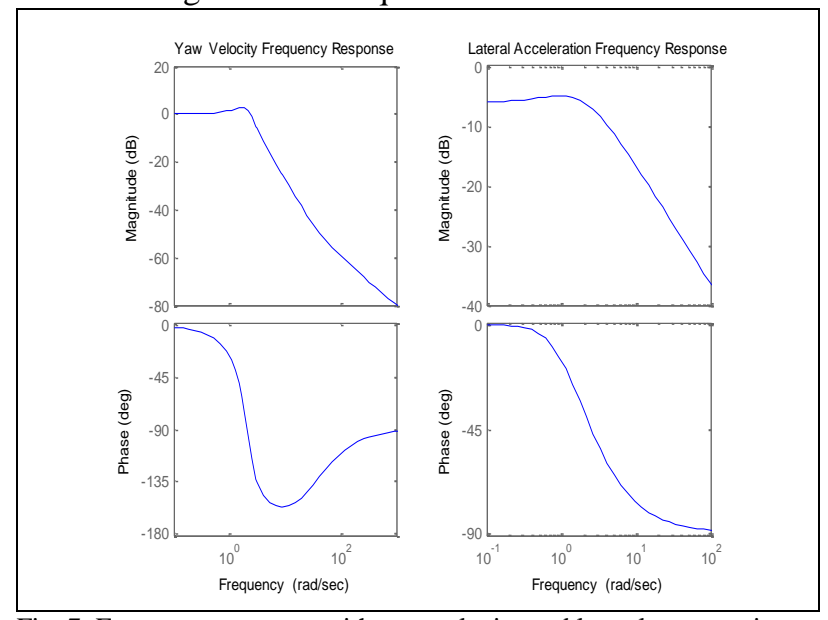

Fig. 7. Frequency response with yaw velocity and lateral accereration

\section{EXAMPLES}

Example 1: The stationary steering behavior of a vehicle is simplified in Fig. 4. The vehicle is moving in a circular orbit with the radius $r=100 \mathrm{~m}$ at a speed of $v=22 \mathrm{~m} / \mathrm{s}$. The following vehicle data are given:

Mass, $m=1,300 \mathrm{~kg}$

Gravity center position, front, $l_{f}=1.2 \mathrm{~m}$ rear, $l_{r}=1.3 \mathrm{~m}$

Lateral tire stiffness, front, $c_{\alpha f}=55,000 \mathrm{~N} / \mathrm{rad}$ rear, $c_{\alpha r}=60,000 \mathrm{~N} / \mathrm{rad}$

(i). Find out the slip angles $\alpha_{f}$ and $\alpha_{r}$, the attitude angle $\beta$, and the steering angle $\delta$.

(ii). Assess the stationary steering behavior of this vehicle.

\section{Solution:}

(i). Moment balance in front point, $F_{L r} l=F_{c e n t} l_{f}$, while $F_{L r}=c_{\alpha r} \alpha_{r}$ and $F_{c e n t}=m \frac{v^{2}}{r}$. Then, $c_{\alpha r} \alpha_{r} l=m \frac{v^{2}}{r} l_{f} \Rightarrow$ $\alpha_{r}=\frac{m v^{2} l_{f}}{r c_{\alpha r} l}$ or

$\alpha_{r}=\frac{1300 \cdot 22^{2} \cdot 1.2}{100 \cdot 60000 \cdot(1.2+1.3)}=0.0503 \mathrm{rad}=2.88^{\circ}$.

Similarly, $\alpha_{f}=\frac{m v^{2} l_{r}}{r c_{l f} l}$ or

$\alpha_{f}=\frac{1300 \cdot 22^{2} \cdot 1.3}{100 \cdot 55000 \cdot(1.2+1.3)}=0.0595 \mathrm{rad}=3.41^{\circ}$.

From equation (9) and (10), $\beta=\alpha_{r}-\frac{\dot{\psi} l_{r}}{v}=\alpha_{r}-\frac{l_{r}}{r}$.

Then, $\beta=0.0503-\frac{1.3}{100}=0.0373 \mathrm{rad}=2.14^{\circ}$.

From equation (9),

$\delta=\alpha_{f}-\beta+\frac{l_{f}}{r}=0.0595-0.0373+\frac{1.2}{100}=0.0342 \mathrm{rad}=1.96^{\circ}$

If the sideslip is ignored, apply the Ackermann condition in Fig. 3, the steering angle then,

$\delta_{\text {Ack }}=\arctan \left(\frac{l}{r}\right)=0.025 \mathrm{rad}=1.43^{\circ}$.

Remarks: The sideslip causes the vehicle movement, $\beta=2.14^{\circ}$, different with the vehicle orientation (steering angle), $\delta=1.96^{\circ}$ and greater than the Ackermann condition, $\delta_{\text {Ack }}=1.43^{\circ}$.

(ii) $\Delta \alpha=3.41^{\circ}-2.88^{\circ}>0 \Rightarrow$ the system is under-steer.

Remark: $\quad \alpha_{f}-\alpha_{r}=\delta-\left(\frac{\dot{\psi} l_{f}}{v}+\frac{\dot{\psi} l_{r}}{v}\right) \quad$ and

$\Delta \alpha=\delta-\frac{l_{f}+l_{r}}{r}=\delta-\frac{l}{r} \Rightarrow \delta=\frac{l}{r}+\Delta \alpha$,

then, $\quad \Delta \alpha=\alpha_{f}-\alpha_{r}=\frac{m v^{2}}{r l}\left(\frac{l_{r}}{c_{\alpha f}}-\frac{l_{f}}{c_{\alpha r}}\right)$

$\delta=\frac{l}{r}+\frac{m}{l}\left(\frac{l_{r}}{c_{\alpha f}}-\frac{l_{f}}{c_{\alpha r}}\right) a_{y}$, when the curve radius $r=$ const, the lateral acceleration, $a_{y}$, increases, the steering angle, $\delta$, must increase as well.

Example 2: 
Examine the yaw natural frequency and the yaw damping of the following vehicle specifications: $l=2.5 m, \quad l_{f}=1.3 m, \quad l_{r}=1.2 m, \quad m=1300 \mathrm{~kg}$, $J_{Z}=1960 \mathrm{kgm}^{2}, \quad c_{\alpha f}=30000 \mathrm{~N} / \mathrm{rad}$. The sideslip stiffness of the rear tires, $c_{\alpha r}$, varies as $c_{\alpha r 1}=30000 \mathrm{~N} / \mathrm{rad} \quad, \quad c_{\alpha r 2}=35000 \mathrm{~N} / \mathrm{rad}, \quad$ and $c_{\alpha r 3}=40000 \mathrm{~N} / \mathrm{rad}$.

\section{Solution:}

The sideslip varies and leads to different steering system. For $\quad c_{\alpha r 1}=30000 \mathrm{~N} / \mathrm{rad} \Rightarrow$ $\left(\frac{l_{r}}{c_{\alpha f}}<\frac{l_{f}}{c_{\alpha r}}\right)=$ oversteer,$\quad c_{\alpha r 2}=35000 \mathrm{~N} / \mathrm{rad} \Rightarrow$ $\left(\frac{l_{r}}{c_{\alpha f}}>\frac{l_{f}}{c_{\alpha r}}\right)=$ understeer, and $c_{\alpha r 3}=40000 \mathrm{~N} / \mathrm{rad} \Rightarrow$ $\left(\frac{l_{r}}{c_{\alpha f}}>\frac{l_{f}}{c_{\alpha r}}\right)=$ understeer as well.

The yaw natural frequency and the yaw damping against the vehicle velocity, $v$, are drawn in Fig. 8.

For over-steer system, there is a critical speed, $v_{\max }=37.8 \mathrm{~m} / \mathrm{s}$, where the vehicle loses the stability and begins to swerve.

For under-steer systems, the yaw damping decreases with the vehicle speed.

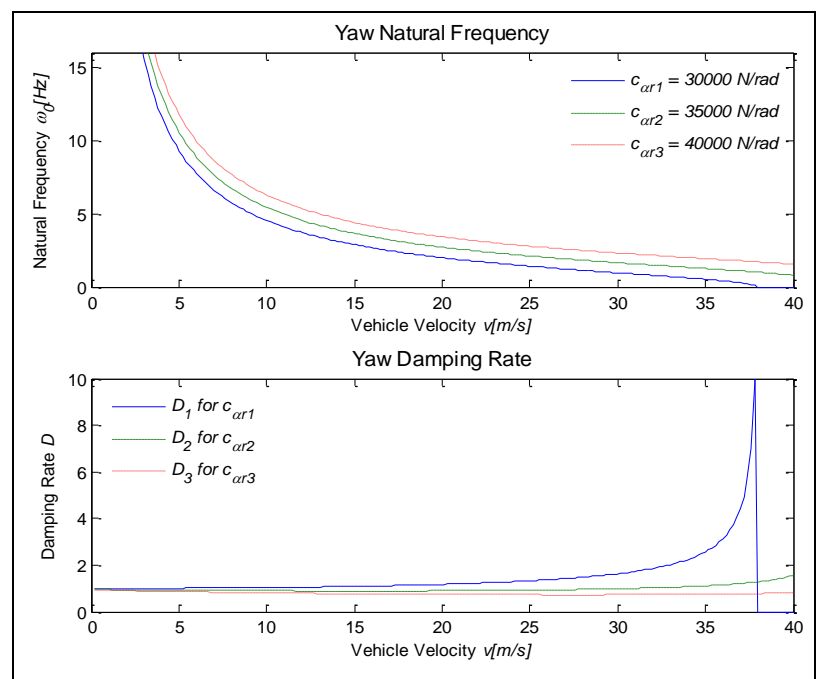

Fig. 8. Yaw natural frequency and yaw damping rate

\section{CONCLUSION}

The single track vehicle model allows analyzing the influences of fundamental parameters such as the effect of the location of the center of gravity, the different front and rear cornering stiffness as well as the understeering/over-steering systems on the vehicle dynamic behavior and sideslip angle. Results from this study can be applied to estimate the tracking errors for an automatic vehicle tracking system in the next step of the project. The analysis of the transient response for this system provides essential knowledge of the vehicle dynamic behaviors under the influences of nonlinear dynamic variables.

\section{REFERENCES}

[1] Kim H.H and Ryu J., "Sideslip Angle Estimation Considering Short-duration Longitudinal Velocity Variation", International Journal of Automotive Technology, Vol 12(4), pp. 545-553, 2011, DOI: 10.1007/s12239-011-0064-2

[2] Hac A, Nichols D., and Sygnarowicz D., "Estimation of Vehicle Roll Angle and Side Slip for Crash Sensing", SAE International Congress, April 2010, Detroit, MI, USA, 2010, DOI: 10.4271/2010-01-0529

[3] Lenain R., Thuilot B., Cariou Ch., and Martinet P., "Mixed Kinematic and Dynamic sideslip angle Observer for accurate Control of Fast Off-road Mobile Robots", Journal of Field Robotics, Vol. 27(2), pp. 181-196, 2010. DOI: 10.1002/rob.20319

[4] Minh V.T., Advanced Vehicle Dynamics, 1st edition, Malaya Press, Pantai Valley, 50603. Kuala Lumpur, Malaysia, 2012, pp. 127-144, ISBN: 978-983-100-544-6

[5] Ackermann J., and Sienel W., "Robust Yaw Damping of Cars with Front and Rear Wheel Steering", IEEE Transactions on Control Systems Technology, Vol. 1(1), pp. 15-20, 1993

[6] Minh V.T and Aziz A.R., "Real-time Control Schemes for Hybrid Vehicle", IEEE International Conference on Control Application (CCA), Denver, CO, USA, September 2011, pp. 538-543, 2011, DOI: $10.1109 / C C A .2011 .6044483$

[7] Minh V.T and Pumwa J., "Simulation and Control of Hybrid Electrolic Vehicle", International Journal of Control, Automation and Systems, Vol 10(2), pp. 308-316, 2012, DOI: $10.1007 / \mathrm{s} 12555-012-0211-1$

[8] Hrgetic M., Deur J and Pavkovic D, "Adaptive EKF-Based Estimator of Sideslip Angle Using Fusion of Inertial Sensors and GPS", SAE International Journal of Passenger Cars - Mechanical Systems, Vol 4(1), pp. 700-712, 2011, DOI: 10.4271/2011-010953 